\title{
Agresividad y habilidades sociales en la adolescencia. Una aproximación conceptual
}

E. Norma Contini ${ }^{1}$

\section{Artículo}

Material original autorizado para la publicación en la revista Psicodebate. Facultad de Ciencias Sociales. Universidad de Palermo.

Recibido 31-03-2015 | Aceptado 15-05-2015

\section{Resumen}

Este artículo tiene como objetivo realizar una revisión del concepto de agresividad, deslindando el solapamiento con otros, tales como agresión, violencia, hostilidad, ira; se enfoca el tema en la etapa adolescencia, por la relevancia que la vinculación con otros cobra en este período, de definición de la identidad; se desarrollan algunos modelos explicativos que se consideran relevantes para la comprensión del fenómeno y que han brindado conceptos para abordar la creciente consulta psicológica al respecto; de entre las teorías se destaca la que conceptualiza a la agresividad como una disfunción de las habilidades sociales, de los modos de relación interpersonal que afectan la salud integral del joven. Se concluye analizando alcances y límites de las teorías y se deja planteada la posibilidad de integración de modelos que permitan obtener una perspectiva multidimensional sobre la agresividad como flagelo en alza en la cultura contemporánea.

Palabras Clave: agresividad, habilidades sociales, adolescentes.

1 Universidad Nacional de Tucumán - Argentina; contini.norma@gmail.com 


\section{Abstract}

The aim of this article is to revise the concept of aggressiveness by defining the overlapping with other concepts, such as aggression, violence, hostility and anger. The subject is focused on adolescence since the relationship to others becomes relevant to defining identity at this stage. Some explanatory models are developed as they are considered important to understand the phenomenon and they provide concepts to approach the growing demand for psychological counselling. Among the theories, we can highlight the one which conceptualises aggressiveness as a dysfunction in social skills, in the interpersonal relationships that affect the adolescent's overall health. Finally, the scope and limits of the theories are analysed and the possibility of integrating models which allow gaining a multidimensional perspective on aggressiveness as a scourge trending upwards in contemporary society is opened up.

Keywords: : aggressiveness, social skills, adolescents. 
El ser humano es gregario por naturaleza. Pasa gran parte de su tiempo en interacción con otros. La vinculación de un modo mutuamente satisfactorio es una fuente de bienestar personal que cobra particular relevancia en la adolescencia. El adolescente se encuentra en la definición de su identidad y en la búsqueda de la pareja, y de allí el valor de las competencias que haya adquirido para relacionarse con los demás. Esto explicaría la importancia que han cobrado los estudios centrados en las habilidades sociales. Las habilidades sociales constituyen un recurso indispensable para la inclusión del sujeto en su grupo de referencia (Gil \& León Rubio, 1998) y puede afirmarse que la salud integral tiene fuertes conexiones con la amplitud y solidez de las redes de apoyo social que se construyen en dicho grupo.

Estos argumentos derivados de numerosos estudios empíricos, en particular en la infancia y en la adolescencia, han puesto en su lugar al desmesurado énfasis que, en el siglo XX adquirieron las habilidades cognitivas, expresadas en medidas sintéticas de Cociente Intelectual (CI). La práctica clínica y psicopedagógica mostró las limitaciones para formular un pronóstico fiable sobre comportamientos eficaces en la vida cotidiana sobre la base de pruebas de CI. Así, fue posible constatar que sujetos con elevado CI tenían dificultades en su desempeño en la vida cotidiana, mientras que otros, de CI discreto lo hacían muy bien. Estos puntos de inconsistencia dispararon la pregunta referida a qué factores entraban en juego para determinar comportamientos eficaces o de fracaso en la vida diaria, que se vinculaban claramente al bienestar personal.

A su vez, existen diversas investigaciones que constatan la existencia de relaciones entre el desarrollo de habilidades sociales en la infancia y adolescencia y el ajuste social, psicológico y académico en la vida adulta (Monjas Casares, 2000; Garaigordobil Landazabal, 2008). Así también muestran una correlación negativa entre habilidades sociales disfuncionales e indicadores de desajustes psicológicos y comportamiento agresivo en la adolescencia (Del Prette, Teodoro, \& Del Prette, 2014).

Más allá de la complejidad del constructo (Contini, 2009), Caballo ofrece una definición que resulta comprensible al señalar que las habilidades sociales son un conjunto de conductas emitidas por un individuo en un contexto interpersonal que expresa sentimientos, actitudes, deseos, opiniones o derechos de ese sujeto de un modo adecuado a la situación, respetando esas conductas en los demás, y que generalmente resuelve los problemas inmediatos mientras minimiza la probabilidad de futuros problemas (Caballo, 2007).

A su vez, mientras las habilidades sociales hacen posible la interacción con pares $\mathrm{y}$ adultos de una manera mutuamente satisfactoria, las disfunciones obstaculizan el vínculo, ya sea por la presencia de agresividad o de retraimiento. La agresividad entre iguales o con los ascendientes en la adolescencia es cada vez más frecuente 
como motivo de consulta, tanto en el campo clínico, educacional o forense. Como señalan Imaz Roncero, Gonzales Gallegos, Geijo Uribe, Higuera González y Sánchez Lorenzo (2013) constituyen una realidad social emergente que ha tomado a los profesionales del campo de la salud sin marcos de actuación sociales y sanitarios preparados para atender este tipo de demandas. Así, constituye un reto poder identificarlas oportunamente, para poder intervenir y modificar tal disfunción.

El objetivo de este trabajo es realizar una revisión del concepto de agresividad, deslindando el solapamiento con otros, tales como agresión, violencia, hostilidad, ira; se desarrollan así también algunos modelos explicativos que se consideran relevantes para pensar la temática; se enfoca la misma en la etapa adolescencial, por la relevancia que la vinculación con otros adquiere en este período de definición de la identidad.

Cabe señalar que el tema ha sido tomado como objeto de estudio en el marco del proyecto de investigación 26-K 504 (aprobado y financiado por la Secretaría de Ciencia, Arte e Innovación Tecnológica de la Universidad Nacional de Tucumán), que se propone la evaluación comparativa de la agresividad en adolescentes de distintos contextos socioeconómicos de Tucumán (Argentina).

\section{Acerca de la agresividad}

Agresividad es una palabra de raíz latina, aggredi que la Real Academia Española, RAE (1992), traduce como provocación o ataque; el sujeto agresivo sería aquel: "propenso a faltar el respeto, a ofender o provocar a los demás" (p. 61). Es decir, la agresividad es una cualidad que se asocia a destrucción o violencia (Imaz Roncero et al, 2013), ya sea hacia sí mismo o hacia los demás (Swann, 2003). Si bien existen numerosas definiciones, estas características están presentes en todas ellas. Diversos autores como Garaigordobil y Oñederra (2010) e Imaz Roncero et al. (2013), utilizan casi de un modo indistinto los términos agresividad y violencia. Lo cierto es que la Organización Mundial de la Salud (OMS) declara en 1996 a la violencia - asociada a la agresividad - como uno de los principales problemas de salud pública en todo el mundo. Desde aquella declaración, el fenómeno ha ido en paulatino ascenso. Así, Osofsky (1999) plantea que en algunos países como EE.UU. es considerada una epidemia y destaca el impacto negativo que tiene en la familia y en la comunidad.

La agresividad comienza a ser conceptualizada a fines del siglo XIX desde enfoques polarizados. Por un lado la Psicología la definió como un instinto, o un impulso innato y, por otro como resultado del aprendizaje. Esta dicotomía, innato-aprendido, tal como afirman Garaigordobil y Oñederra (2010) ha sido una constante en los estudios psicológicos sobre agresividad y, en la actualidad, se 
observa un giro conceptual, que considera interdependientes a múltiples factores, tales como los personales, familiares, educativos y sociales.

Si bien para algunas teorías -como se verá más adelante - la agresividad es imprescindible para la supervivencia de los seres vivos, en el campo de la psiquiatría y psicología se presenta asociada a diversos cuadros psicopatológicos. Así, la Clasificación Internacional de Enfermedades de la OMS -CIE 10(Organización Mundial de la Salud, 1999), en el apartado destinado a los trastornos de inicio en la infancia y adolescencia incluye las categorías Trastornos disociales y Trastorno del comportamiento social de comienzo en la infancia y adolescencia. Cabe destacar que, en la descripción del primero de estos coexisten los constructos agresividad y violencia.

Por su parte el Manual Diagnóstico y Estadístico de las Enfermedades Mentales -DSM 5- (American Psychiatric Association, 2014) incluye las siguientes categorías diagnósticas: Trastornos Destructivos, del control de los impulsos $y$ de la conducta, que contiene como subcategorías al Trastorno negativista desafiante, al Trastorno explosivo intermitente y al Trastorno de la conducta, en todos los cuales están descriptos comportamientos agresivos y vinculados con situaciones de violencia. Igualmente se aclara que pueden tener comienzo en la infancia y en la adolescencia. Por último, se incluye en la categoría Trastornos de la Personalidad tipo B, a la subcategoría Trastorno de la Personalidad antisocial, que se registra a partir de los 15 años.

En la literatura el constructo agresividad se presenta asociado con otros, como agresión, violencia, hostilidad, conducta antisocial, y ello hace compleja su definición y clasificación. La agresividad implica comportamientos con manifestaciones fenomenológicas distintas, con funciones también diferentes, que pueden estar determinadas por diversas circunstancias externas (Andreu, Ramírez \& Raine, 2006) y por mecanismos genéticos distintos.

A diferencia de la agresión, que constituye un comportamiento específico, reactivo frente a situaciones concretas, la agresividad es una disposición a actuar en distintas situaciones, atacando física o verbalmente a otro, o a ofender de un modo intencional (Carrasco Ortiz \& González Calderón, 2006). La ira, en cambio constituye un estado emocional que puede presentarse como un ligero enojo hasta una furia intensa, y no está dirigida a una meta (Spielberger, Jacobs, Russell \& Crane, 1983). Mientras que la hostilidad tiene la connotación de actitudes negativas que motivan comportamientos agresivos dirigidos a dañar objetos o personas (Spielberger et al, 1983). La hostilidad representaría el componente cognitivo, mientras que la ira, el emocional. En función del solapamiento de los tres términos, Spielberger los empleó en forma conjunta como Síndrome AHA, aunque como se los ha definido precedentemente, no son intercambiables. 
Con relación al solapamiento entre los conceptos de agresividad y violencia, la violencia según la RAE (1992) implica: "aplicar medios violentos a cosas o personas para vencer su resistencia"... "se trata de alguien que "obra con ímpetu y fuerza extraordinarias... que se hace bruscamente" (p. 2093). Como bien señalan Carrasco Ortiz y González Calderón (2006) algunas definiciones de violencia no se diferencian de las de agresividad, pero sí se destaca que en algunas definiciones de violencia se hace referencia a la amenaza o intimidación que no siempre están incluidas en las conceptualizaciones de agresividad. El concepto de violencia refiere a comportamientos agresivos cuya intensidad y destructividad parecieran ser mayores que las observadas en un acto agresivo; otra característica es la aparente carencia de justificación. En la literatura se plantea que estas características no son definitorias de la agresividad, y que la respuesta agresiva puede, en cambio, ser legítima como forma de responder a un ataque externo (Carrasco Ortiz \& González Calderón, 2006). Se considera que estas disquisiciones son polémicas ya que, desde un punto de vista clínico, puede afirmarse que los comportamientos responden a causas, no carecen de justificación y, por otro que el ingreso a la cultura implica el aprendizaje de formas de afrontar la adversidad (ataque externo) por vías no agresivas. Dadas estas dificultades, ambos términos se presentan en la literatura solapados o empleados como sinónimos.

\section{Algunas clasificaciones de la agresividad}

En función de la complejidad del constructo y diversidad de expresiones del comportamiento agresivo, se han intentado múltiples clasificaciones. Así, la Clasificación Internacional de Enfermedades de la OMS -CIE 10- (Organización Mundial de la Salud, 1999) los divide en: agresiones socializadas (holgazanear, apropiarse de dinero a escondidas, consumo de alcohol y drogas, estafas) y no socializadas (peleas, intimidaciones, alteraciones y explosiones). Otra categorización distingue entre agresividad impulsiva y agresividad premeditada. Halsband y Barenbaum (2008) describen a la agresividad impulsiva como una reacción rápida ante un estímulo percibido como una amenaza u ofensa. Está motivada por la ira o por el miedo y el sujeto no logra prever las consecuencias. Suele ir acompañada por un aumento de la presión arterial, de frecuencia cardíaca o rubor, siendo la descarga un fin en sí mismo. En cambio, la agresividad premeditada se propone obtener un fin específico. No se trata de una reacción espontánea, sino previamente pensada o calculada, tampoco va acompañada de cambios somáticos. El sujeto impresiona como tranquilo, y su accionar puede ser mucho más peligroso que el tipo descripto previamente.

Como señalan Halsband y Barenbaum (2008), la agresividad puede considerarse normal o patológica y en esa valoración inciden patrones culturales. Esta relatividad cultural le otorga mayor complejidad al estudio de este tema. 
En tal sentido Buss (1961) y Buss \& Perry (1992) categorizaron la agresión en: a) físico-verbal, b) activa-pasiva, c) directa-indirecta.

En la dimensión físico-verbal se diferencia entre el uso de la fuerza o del lenguaje para infringir daño a un sujeto. La dimensión activo-pasiva alude al modo en que el agresor se involucra en la generación de daño a otro. Un modo activo sería por ejemplo que un adolescente se ofusque con el profesor que lo desaprobó - de un modo injusto según su perspectiva -; el adolescente procede entonces a estropear los neumáticos del automóvil del docente. Una forma pasiva de agresividad sería que el adolescente estuviera acompañado por un amigo, quien es testigo de la acción, pero no interviene señalando lo inadecuado del proceder de su par.

La dimensión directa-indirecta: en la directa, la acción de un sujeto tiene como objetivo central producir un daño en forma directa a otra persona. Dicha agresión puede ser verbal o física, por ejemplo, golpear a alguien o gritarle. En cambio, la agresión indirecta tiene también el mismo propósito, pero se realiza por medio de otras personas o pertenencias, por ejemplo, dañar la propiedad de una persona, o hablar mal de él "a sus espaldas".

\section{Algunas teorias explicativas de la agresividad}

La agresividad como fenómeno presente en diversas culturas y como un tema central en el campo de la salud mental ha sido objeto de numerosos estudios que han buscado mayor claridad en la definición del constructo y que se han propuesto generar teorías explicativas. Dichas teorías son las que, según su potencia, brindan insumos para diagramar intervenciones que promuevan el cambio de la agresividad al buen trato. En la revisión bibliográfica se registran variadas teorías: biológicas, del aprendizaje social, psicoanalítica, de la frustración-agresión, bifactorial, de las habilidades sociales, contextuales.

En este trabajo se desarrollan solo algunas, tomando como criterio las que han puesto el foco en infancia y adolescencia, o bien que han contribuido a la comprensión del fenómeno para poder operacionalizarlo en pruebas que permitan su evaluación. No se desarrollan teorías de la envergadura de la psicoanalítica, por existir abundante bibliografía, que no podría ser recreada con la profundidad necesaria, en el marco de las limitaciones de la extensión de este artículo.

\section{Teorías biológicas}

Se hará referencia a dos desarrollos que se consideran destacables, los estudios de Lorenz y las investigaciones contemporáneas centradas en el funcionamiento bioquímico. 
Konrad Lorenz, médico y zoólogo austríaco y premio Nobel de Medicina en 1973, fundó la Escuela etológica de Tinbergen e hizo sustanciales contribuciones para conocer el comportamiento animal y su papel en el proceso de adaptación y supervivencia. Hace referencia al instinto que tienen en común los animales y el hombre: el instinto de la agresión. De su vasta obra se destaca el libro La agresión: el pretendido mal (Lorenz, 1998). Se interroga por qué y para qué luchan entre sí los animales de la misma especie, y también los seres humanos. Con sus investigaciones trata de dar respuestas a la pregunta sobre cuáles son las causas de la agresión entre los sujetos que constituyen una amenaza de aniquilamiento para la humanidad, y si el conocimiento de tales causas puede orientar la acción para modificar esta dramática situación.

La tesis central de Lorenz $(1971,1998)$ es que la agresión es un instinto. Define al instinto como un mecanismo innato, que hace posible un comportamiento, que tiene bases biológicas, su origen en la evolución filogenética y que se transmite hereditariamente.

Lorenz emplea el concepto de agresión intraespecifica, que determinaría una amplia gama de comportamientos destructivos. En el pensamiento de Lorenz ha influido la teoría de Darwin, que afirma que las formas superiores de vida han surgido a partir de la evolución de otras inferiores. La lucha intraespecífica ha determinado que sobrevivan las especies mejor dotadas. En esa línea de pensamiento, el instinto agresivo ha surgido en el curso de la evolución. Ofrece numerosos ejemplos de estudios con animales -en los que es experto - y plantea que la agresividad no es un fenómeno intrínsecamente malo, sino que cumple una función esencial en la conservación de la especie. De la observación de peces de coral y de estudios experimentales con ratas, detalla diversas funciones de la agresión que pueden sintetizase en:

a) El cambio de conducta de sociable a agresiva cuando las ratas se encontraban con otras que no pertenecían a la misma comunidad.

b) Territorialidad: la agresividad se desencadena al acercarse un animal de la misma especie al territorio del otro.

c) Defensa de los hijos: analiza cómo la agresividad aparece con relación a la defensa de aquellos: en los animales en los cuales uno de ellos está destinado al cuidado de la cría, sólo éste es potencialmente agresivo con sus congéneres.

d) Establecimiento de una jerarquía dentro de la sociedad animal organizada. Lorenz (1998) pudo comprobar que cada uno de los integrantes que vivía en una sociedad sabía identificar quien era más fuerte que él, o más débil. A partir de este reconocimiento el más débil se retira sin presentar comportamientos de lucha, mientras que el más fuerte tiene la expectativa de que el más débil se aleje si lo encuentra en su camino. 
e) La agresividad hace posible la emergencia de vínculos amistosos entre lo sujetos. Para llegar a esta tesis, analiza que aquellos animales con instinto de agresión muy bajo, solo eran capaces de formar lo que Lorenz denominó multitudes anónimas, o sea masas de individuos en los que no existía ningún tipo de identificación, ni de diferenciación entre uno y otro. Caracteriza a esta multitud el hecho de que los individuos de una misma especie reaccionan unos sobre otros por atracción mutua, y se unen mediante pautas de comportamiento que uno o algunos integrantes desencadenan en los demás.

Observó que el vínculo personal aumentaba en la medida que se incrementaba también el instinto agresivo. Según Lorenz, la construcción de la amistad iría unida en sus orígenes a la agresión intraespecífica. Si se analiza este concepto, en realidad lo que Lorenz designa como agresividad sería una pulsión vital que orienta la vinculación entre lo sujetos y, por tanto, desprovista de toda connotación patológica. Señala: "no sabemos en cuántos y hasta qué punto importantes modos de comportamiento humano entra la agresión como factor motivante, pero opino que deben ser muchos..." (Lorenz, 1998, p. 313). Alude al aggredi, traducido como gradus, o sea paso y ad, hacia, que significaría avanzar, ir hacia adelante) que, en su sentido original, significa afrontar situaciones que son vitales para la supervivencia. Para Lorenz, a partir del establecimiento del lazo de amistad, la agresividad de un individuo deja de infligirse sobre el otro, pero se ejercita sin inhibiciones sobre todos los otros individuos de las denominadas multitudes anónimas. Aquí se establecerían las categorías amigo y extraño en los vínculos entre individuos.

Lorenz ha tratado de hacer transferencia de los resultados de sus investigaciones del ámbito animal al humano. De este modo considera que la agresión intraespecífica no tiene una connotación negativa, sino que es parte esencial de la conservación de la vida, aunque admite que en ocasiones puede operar de un modo disfuncional y ser destructiva. Considera que la agresión puede convertirse en peligrosa en el ser humano - a diferencia del animal - por la aparición del pensamiento conceptual. Dicho pensamiento le ha permitido un rápido progreso, como el descubrimiento del fuego o la fabricación de armas para defenderse. Sostiene que dicho progreso no se ha visto acompañado de una adaptación del instinto agresivo a esa nueva situación; los hombres comenzaron a emplear dichos instrumentos para eliminar a sus pares. Por otra parte, a partir del empleo de los medios técnicos que fue creando se volvió capaz de dominar el medio exterior, evitando de este modo el peligro que de allí provenía. Este sería el segundo motivo del riesgo del instinto agresivo en los seres humanos. La tesis de este autor es que cuando un instinto deja de cumplir una función de conservación 
de una especie sobre otra especie, puede volverse estéril y, en el caso de la agresión puede convertirse, inclusive, en peligroso.

Frente a este planteo, pesimista por cierto, considera que es posible evitar que la agresividad se convierta en peligrosa, a partir de la reorientación hacia objetos sustitutivos. Otra posibilidad sería mediante lo que en la teoría psicoanalítica se denominó sublimación. La sublimación es un concepto propuesto por Freud para explicar ciertas actividades del hombre que aparentemente no guardarían relación con la sexualidad, pero que encontrarían su energía en la fuerza de la pulsión sexual. Menciona así actividades como el arte y la investigación intelectual. En suma, se dice que la pulsión se sublima, en la medida en que es derivada hacia un nuevo fin no sexual, y se orienta a objetos socialmente valorados (Laplanche \& Pontalis, 2009). La sublimación sería por lo tanto eficaz para calmar la tensión producida por la inhibición de las pulsiones agresivas. Lorenz igualmente sostiene como muy valioso el establecimiento de vínculos interpersonales entre miembros de una familia o de grupos diferentes. Finalmente, atribuye mayor importancia a la canalización del entusiasmo militante, de un modo inteligente, es decir, contribuyendo a que las generaciones más jóvenes encuentren en el mundo que les toca vivir causas dignas de asumirse.

Si esta reorientación ocurre, la agresividad continuará cumpliendo importantes funciones, pero se verá complementada por mecanismos inhibidores que impidan que la agresión se ejercite en forma directa y destructiva entre congéneres.

Las críticas a la tesis de Lorenz apuntan a que hace extrapolaciones del comportamiento animal al comportamiento humano; que otorga un valor determinante al instinto agresivo en el comportamiento, sin tener en cuenta el papel del pensamiento, la posibilidad de razonar, de simbolizar, propios de la condición humana, y que marcan una sustancial diferencia con los animales.

\section{Acerca de las bases biológicas de la agresividad}

Las teorías biológicas desde hace mucho tiempo han buscado la relación entre áreas cerebrales y la agresividad y, con el gigantesco desarrollo contemporáneo de la bioquímica, la genética, la microbiología y la imagenología, se viene investigando acerca de la relación entre la agresividad y los déficit genéticos (a nivel de cromosomas), hormonales y de los neurotransmisores (Garaigordobil \& Oñederra, 2010; Halsband, et al., 2008).

Con relación a las áreas cerebrales que serían responsables del comportamiento agresivo, se pasó de una primera etapa localizacionista a otra que pone énfasis en circuitos cerebrales complejos que abarcan varias áreas y que se presentan vinculados con el comportamiento agresivo (Davidson, Putnam \& Larson, 2000). Así, se ha profundizado el estudio del papel de la corteza orbitofrontal, de la 
amígdala, el hipotálamo y el hipocampo, entre otros. En estudios experimentales con animales, el hipocampo es el que presenta una mayor relación con la agresividad.

Los estudios actuales hacen referencia a la fisiopatología de la agresividad encontrándose relación entre los neurotransmisores y la agresividad. Así, se plantea que: "en términos generales, puede decirse que en nuestra especie los circuitos catecolaminérgicos, al igual que los andrógenos, estimulan la agresividad, mientras los serotonérgicos tienden a inhibirla" (Halsband \& Barenbaum, 2008, p.23). En suma, se trata de un campo en creciente expansión por el apoyo tecnológico con el que cuentan las investigaciones. Así también, en la literatura especializada se observa una búsqueda de vinculación entre disfunciones de base biológica y fármacos con potenciales efectos terapéuticos. Al igual que lo planteado para la teoría de Lorenz, este enfoque sería reduccionista, al encontrar como causa central de la agresividad alteraciones de orden genético y de neurotransmisores. Sin embargo, resultan cautelosas las conclusiones a las que arriban Halsband y Barenbaum al afirmar que, sobre los mecanismos productores de la agresividad, es más lo que se ignora que lo que se conoce.

\section{La teoría bifactorial}

Uno de los estudios más significativos del comportamiento social en niños es el realizado por Kohn (1977) de revisión de investigaciones efectuadas entre 1920 y 1960. Ello le permitió enunciar la teoría bifactorial que afirma la existencia de dos factores bien definidos en dicho comportamiento social: retraimiento y agresividad-conducta antisocial. Como señala con acierto Kohn, esta regularidad es poco frecuente en el campo de las ciencias sociales.

En esta línea los estudios de Achenbach y Edelbrock (Achenbach, 2008; Achenbach \& Edelbrock, 1979) y Silva, Moro y Generós Ortet (1997) han producido un avance conceptual relevante impactando en la construcción de instrumentos de evaluación específicos.

Achebanch \& Edelbrock también propusieron una clasificación de dos grandes factores en el comportamiento social, que denominaron de primer orden y factores de segundo orden, menos amplios. Encontraron un primer gran factor bipolar que denominaron internalización / externalización. Este gran factor presenta una elevada semejanza con los dos factores de la teoría bifactorial de Kohn (Martorell, 1997). Encontraron, a su vez, un segundo factor que designaron como de patología difusa, indicador de psicopatología más severa.

Por su parte, un modelo contemporáneo que resulta clínicamente útil para analizar las habilidades sociales es el denominado Modelo del Aspa (Silva et al., 1997). Los autores prefieren emplear el término orientación personal positiva y 
negativa, antes que comportamiento social, por entender que este constructo es demasiado amplio y ambiguo.

Silva et al. (1997) proponen dos grandes dimensiones bipolares, a) conducta prosocial-conducta antisocial y b) alta sociabilidad- baja sociabilidad. En sus investigaciones identificaron que la baja sociabilidad se asociaba con la ansiedad social, timidez y retraimiento (que corresponde al factor internalización en el modelo de Achenbach) mientras que el comportamiento antisocial aparecía vinculado a agresividad, obstinación, oposición a normas y a comportamientos delictivos (factor externalización en el modelo de Achenbach).

Es en la adolescencia cuando comienzan o se incrementan los problemas externalizantes -conducta antisocial, agresividad, violencia- e internalizantes -timidez, ansiedad social- (Inglés et al., 2009). Estos comportamientos disfuncionales implican grandes costos sociales, económicos, familiares e individuales, por lo que merecen ser investigados en profundidad.

\section{Teoría de la frustración-agresión}

Como representantes de esta teoría se destacan Dollard, Doob, Miller, Mowerer y Sears (1939), quienes plantean que el comportamiento agresivo surge cuando el sujeto percibe bloqueadas o interferidas las posibilidades de acceder a una meta. Las situaciones de privación no inducen siempre al comportamiento agresivo, sino que éste se presenta cuando el sujeto no logra satisfacer un logro deseado. En tal sentido, la tolerancia a la frustración pareciera ser un factor primordial en la adaptación del sujeto a su entorno. Señala que la intensidad de la agresividad es directamente proporcional a la intensidad de la frustración. La frustración predispondría a la agresividad, pero bajo determinadas condiciones.

Desde este modelo se concluye que la no satisfacción de las necesidades básicas llevaría a los seres humanos, al igual que a los animales, a desarrollar comportamientos agresivos. En esta línea, Dollard et al. (1939) plantean que: "la agresión es siempre una consecuencia de la frustración [...] la frustración siempre lleva a alguna forma de agresión" (p. 1).

Se distinguen dos tipos de comportamiento: la agresividad emocional y la agresividad instrumental. La agresividad emocional se produce por la falta de logros frente a una situación esperada; la agresividad instrumental sería un comportamiento aprendido, aceptado o valorado socialmente, por lo cual no necesariamente emerge de una contrariedad (frustración). 


\section{Déficit en el procesamiento de la información}

Los desarrollos de la Psicología Cognitiva hicieron posible a partir de los ' 80 analizar el comportamiento agresivo desde la perspectiva del procesamiento de la información. Los estudios realizados concluyen en que el comportamiento agresivo surge como consecuencia de un déficit en el procesamiento de la información. Tal distorsión conduce a un afrontamiento ineficaz de los problemas de la vida cotidiana (Dodge \& Crick, 1990).

Desde este modelo, en el sujeto se encuentren alojadas particulares estructuras de memoria socio-cognitivas o guiones que brindan insumos para lograr un procesamiento específico de solución de problemas. Los sujetos agresivos presentarían procesos erróneos en la búsqueda de estrategias adecuadas o en la disponibilidad de información en la memoria a largo plazo. Tienden a codificar inadecuadamente las situaciones sociales y las señales internas (Pakaslahti, 2000), a interpretar y analizar las situaciones más sobre la base de hechos pasados que a partir de las circunstancias concretas que les corresponde resolver en el momento presente. Como consecuencia de ello tienden a generar estrategias agresivas para resolver los problemas.

\section{Teoría de las habilidades sociales}

Desde esta teoría se sostiene que el comportamiento agresivo deriva de inadecuadas competencias sociales. Toma conceptos de la teoría del déficit en el procesamiento de la información social desarrollada precedentemente. Tal déficit conduce a los sujetos a una errónea interpretación de las señales sociales que emite su interlocutor.

Se ha podido comprobar que las habilidades sociales de agresores y víctimas en la infancia - como en la adolescencia - tienen una cualidad distinta y que los agresores puntúan más alto en las pruebas que evalúan dichas habilidades (Garaigordobil \& Oñederra, 2010). En tal sentido, se ha encontrado como propio del perfil del agresor la desinhibición, la ausencia de temor al rechazo, el deseo de dominio y un rasgo central, la capacidad de manipulación en la vinculación con otro.

En los estudios realizados con niños agresivos se destaca la falta de empatía, esto es, la dificultad para adoptar la perspectiva de los demás (Garaigordobil \& Oñederra, 2010). Los niños agresivos suelen interpretar de un modo erróneo las señales sociales que emite su interlocutor, decodifican estímulos ambiguos, como si fueran agresivos. Por ejemplo, un niño interpreta durante el recreo en el colegio que dos compañeros que están a cierta distancia espacial de él se encuentran criticándolo - por la forma en que lo miran - y, por tal motivo, se rehúsan a que 
se lo incluya en la conversación; esta interpretación errónea genera una respuesta, que se expresa en un insulto hacia sus pares. Tal comportamiento agresivo va a activar una reacción hostil en aquellos. Crick y Dodge (1996) afirman que ese sistema de atribución es más frecuente en niños agresivos y que, por otra parte, en la toma de decisiones prefieren asumir comportamientos agresivos por valorarlos como más eficaces para lograr los objetivos que se proponen.

\section{Las teorías interactivas y del aprendizaje social}

Más allá de la diversidad de conceptualizaciones sobre las habilidades sociales y sobre la agresividad como una de sus disfunciones, la mayoría de los investigadores acuerda que en las mismas están implicados factores individuales, de contexto y, esencialmente, la interacción entre ambos. De ello se deduce que los denominados modelos interactivos son los que permiten comprender más cabalmente cómo y por qué un sujeto adquiere determinadas habilidades sociales.

De entre de estos, dos resultan de interés: el modelo interactivo propiamente dicho y el del aprendizaje social.

Según el modelo interactivo, las habilidades sociales serían el resultado de procesos cognitivos y de comportamiento en una secuencia que se inicia con la percepción correcta de estímulos interpersonales relevantes, continúa con el procesamiento flexible de estos estímulos para evaluar la mejor opción de respuesta, y concluye con la manifestación de la opción elegida (León Rubio \& Medina Anzano, 1998). El sujeto tiene en este modelo un rol activo, en cuanto busca, procesa información y controla sus acciones. La característica esencial es la influencia mutua entre las dos personas que interactúan. Trower (1986) y McFall (1982) son dos figuras significativas dentro de este modelo.

\section{Los aportes de Bandura}

Bandura, destacado psicólogo canadiense, hace un aporte sustancial a la comprensión del comportamiento agresivo.

Sobre la base de rigurosos estudios experimentales, desarrolla una serie de principios del aprendizaje, y pone mucho más énfasis en las variables sociales que otras teorías ya existentes. Así es que denomina a su enfoque como sociocomportamental (Bandura \& Walters, 1974).

El interés de Bandura es poder explicar la adquisición de nuevas comportamientos sociales, en nuestro particular caso, el interés por conocer cómo se adquiere el comportamiento agresivo, para lo cual hace investigaciones sobre el papel de la imitación, el aprendizaje por observación, las pautas de refuerzo (o recompensa) en la adquisición de comportamientos socialmente aceptables o 
censurados, como la agresividad y sobre la autorregulación y la autoeficacia.

Con relación a la imitación, señala que ésta es un aspecto esencial del aprendizaje. Bandura reconoce a Miller y Dollard como estudiosos del papel de la imitación en el aprendizaje, pero avanza señalando que existen pruebas de que se puede aprender por observación del comportamiento de otro, aun cuando el sujeto que observa no reproduzca el modelo de comportamiento durante el periodo de adquisición, es decir que no haya recibido refuerzos.

Al igual que Skinner admite que gran parte del aprendizaje ocurre por refuerzo, pero se diferencia de aquél al afirmar que casi todos los comportamientos se pueden adquirir sin él, es decir por observación. Propone así el nombre de reforzamiento vicario, para plantear que el sujeto observa el comportamiento de otro y las consecuencias que esa conducta implica. Agrega que en diversas culturas los niños no hacen lo que los adultos les dicen que hagan, sino más bien lo que les ven hacer. Aquí radica la sustancial diferencia con el conductismo, al argumentar que no sólo se aprende por experiencia directa. Otra diferencia con Skinner radica en que Bandura atribuye mucho valor a los procesos cognitivos en el aprendizaje por observación. El sujeto, en nuestro caso, el adolescente, no imita mecánicamente los comportamientos que observa, sino que analiza y prevé las consecuencias de los mismos. Los procesos cognitivos median entre estímulo y respuesta y no existe entonces una conexión directa entre ambos, ni entre comportamiento y reforzador, como supuso Skinner (Schultz \& Schultz, 2010).

También estudió el papel del autorreforzamiento, al que define como: "administrarse uno mismo recompensas o castigos por cumplir, superar o incumplir las expectativas o normas personales" (Schultz \& Schultz, 2010, p. 408). Esta teoría se apoya en la noción de agenciar, en la cual el sujeto tiene la función de anticipación y de autoevaluación de los reguladores proactivos de sus motivaciones y acciones (Bandura, 2001; Bandura \& Locke, 2003). Difiere de otras teorías que solo se sustancian en el control del feed back negativo cuyo propósito es la corrección del error.

Para Bandura el autorreforzamiento es tan importante como el reforzamiento ejercido por otros. Por ejemplo, con relación al comportamiento agresivo, un adolescente, que en una reunión social agredió verbal y físicamente a un amigo por un entredicho con relación a una joven expresará, "no iré más a fiestas de quince años mientras no me controle; no puedo hacer este mal papel'". Expresa de este modo un autocastigo.

A su vez, el logro de los comportamientos deseados por el sujeto incidirá en su autoeficacia. Para Bandura, la autoeficacia está compuesta por los sentimientos de adecuación, eficiencia y competencia para asumir las situaciones que la vida plantea. También podría definirse como creer que uno puede. Esa vivencia de autoeficacia se mantiene o eleva en la medida que el sujeto logra el desempeño 
que necesita o desea. Bandura también refiere a la autoeficacia a partir de la noción del control que el sujeto ejerce sobre su propia vida.

\section{Acerca del modelado. Los estudios sobre agresividad}

Para fundamentar su teoría Bandura se apoya en rigurosas investigaciones. Una de las más conocidas es la iniciada por su discípulo Walters, denominada "el muñeco bobo". Se trata de un estudio empírico con niños preescolares a quienes se les presentó una filmación, en la cual un adulto infligía golpes y agredía a un muñeco inflable; a continuación se dejó solos a los niños en una sala con un objeto semejante y se constató que habían "modelado" su comportamiento de acuerdo con lo que acababan de visualizar en el film. No solo repitieron las agresiones que habían observado que el adulto profería, sino que agregaron otras acciones y verbalizaciones igualmente agresivas. Se comparó el comportamiento de los niños con un grupo control (no habían visualizado el film con el modelo de interacción agresiva), constatándose que el grupo experimental había manifestado el doble de agresividad que el grupo control.

Sobre la base de estos resultados Bandura afirma que el aprendizaje ocurre no solo por imitación, sino por observación y que se puede acelerar por la presencia de modelos, a los cuales no imita mecánicamente, sino que infiere reglas y consecuencias del comportamiento de aquellos. En síntesis, observando el comportamiento de un modelo e imitándolo, el sujeto puede adquirir respuestas que no tenía, o bien se pueden fortalecer o debilitar las que tiene actualmente (Schultz $\&$ Schultz, 2010). Esta teoría otorga así un elevado peso al contexto. Con relación a la agresividad, cabe reflexionar sobre los modelos que brindan los mass media en el contexto cultural actual, y las dificultades que puede encontrar un adolescente para vincularse de un modo satisfactorio con otros, dado el choque de modelos familiares con los que proporcionan los medios masivos de comunicación.

A modo de síntesis cabe decir que la teoría de Bandura ha sido ampliamente aceptada por su potencia explicativa; prueba de ello es la vasta producción de libros y artículos de investigaciones sobre la base de sus principios.

Las críticas plantean que esta teoría opera a nivel del comportamiento manifiesto, dejando de lado emociones y conflictos humanos; a su vez, no ha logrado explicar de qué modo las variables cognitivas afectan dicho comportamiento, en nuestro caso, el comportamiento agresivo.

\section{Discusión}

Se ha tomado como objeto de estudio la agresividad, con especial referencia a la adolescencia, con la modalidad de artículo de revisión por tratarse de un tema cada vez más frecuente en la consulta psicológica; vinculada a la violencia, la 
agresividad se presenta como un flagelo en la cultura contemporánea, y, como bien ha señalado Lorenz constituye una amenaza de aniquilamiento para la humanidad.

En la conceptualización de la agresividad se analizó el solapamiento con otros constructos. De todos ellos, se retoma el de la violencia, por sus implicancias en el campo de la salud integral del adolescente. Se dijo así que la violencia refiere a comportamientos agresivos cuya intensidad y destructividad parecieran ser mayores que las observadas en un acto agresivo y presentan una aparente carencia de justificación. Estas características parecieran no ser definitorias de la agresividad; se planteó en cambio que el comportamiento agresivo puede llegar a ser legítimo como forma de respuesta a un ataque externo. Estas disquisiciones son polémicas, ya que desde un punto de vista clínico puede afirmarse que los comportamientos responden a causas, no carecen de justificación y, por otro, que el ingreso a la cultura implica el aprendizaje de formas de afrontar la adversidad (ataque externo) por vías no agresivas. No es por azar, entonces que, en las prácticas psicológicas, ambos términos se presenten asociados.

Cabe destacar que las definiciones acerca de la normalidad o patología de la agresividad se ven fuertemente impactadas por la cultura, pero más allá de este relativismo cultural, se puede afirmar que la agresividad es un comportamiento que se presenta asociado con diversos cuadros psicopatológicos, tanto en la adolescencia como en otras etapas del ciclo vital; es por ello que no sería prudente considerar a la agresividad como una respuesta esperable en un sujeto con buen ajuste emocional.

Entre las teorías consideradas se destaca la polaridad entre las posturas biológicas y las contextuales por un lado, y por otro si puede ser considerado un comportamiento normal o patológico.

De las teorías biológicas destacan los estudios de Lorenz, quien a partir de minuciosas investigaciones del comportamiento animal concluye que la agresión es un instinto que comparte con el hombre. Su pregunta acerca de por qué y para qué luchan entre sí los animales de la misma especie, y también los seres humanos mantiene absoluta vigencia hoy. La tesis de Lorenz sitúa a la agresividad en el plano de un mecanismo innato, que no sería intrínsecamente malo, que tiene bases biológicas y está destinado a la conservación de la especie. Pero Lorenz avanza al plantear que la agresividad, entendida como ir hacia adelante, avanzar, hace posible el vínculo amistoso entre los seres humanos. Se observa aquí un cambio de posición, ya que no se trataría de la agresividad, como acometer a otro, sino como avanzar en la búsqueda de un par, concepto también presente en la teoría de las habilidades sociales. Según entiende Lorenz, a partir del establecimiento de un lazo de amistad entre congéneres, la agresividad de un sujeto deja de infligirse sobre otro. Inaugura el concepto de multitudes anónimas, donde sitúa a aquellos animales con bajo instinto de agresión, característica que les impediría hacer un 
lazo con otros. Es probable que se pueda pensar estas dos configuraciones del aggredi, desde los estudios contemporáneos de Kohn; Kohn, formula la teoría bifactorial, al encontrar una regularidad poco frecuente en la estudios psicológicos en la infancia, en comportamientos que denominó Internalizantes (serían los de bajo instinto de agresión de Lorenz) y Externalizantes, los de un nivel más elevado de instinto agresivo. Cuando la polaridad externalizante se presenta muy acentuada, se estaría en presencia del comportamiento agresivo. Lorenz advierte que la agresión puede convertirse en peligrosa en el ser humano - a diferencia del animal - por la aparición del pensamiento conceptual. Dicho pensamiento le ha permitido un rápido progreso, como el descubrimiento del fuego o la fabricación de armas para defenderse, pero no se ha visto acompañado de una adaptación del instinto agresivo a esa nueva situación; los hombres comenzaron a emplear dichos instrumentos para eliminar a sus pares y también para dominar el medio exterior. Con ello se convertiría en estéril y peligroso. El planteo de la potencial reorientación de dicho instinto hacia otros fines socialmente aceptables conecta con el planteamiento de la sublimación de la teoría freudiana.

La teoría de Lorenz presenta la limitación de hacer transferencia de resultados de la etología a la condición humana, desconociendo la dimensión simbólica, la posibilidad de pensar privativa del ser humano. Desde una perspectiva psicológica, la pregunta gira en torno a qué circunstancias inherentes a la cultura generan una disfunción en esa capacidad de simbolizar, capacidad que debiera operar como freno a ese acometer del comportamiento agresivo. En tal sentido es también llamativo que en los estudios con animales se hace referencia a agresividad, mientras que en los estudios con humanos aparece vinculado a otro concepto, la violencia, que adquiere mayores connotaciones psicopatológicas.

Desde una perspectiva biológica los estudios actuales se han visto fuertemente potenciados por el avance de la tecnología biomédica. Se ha puesto énfasis en la fisiopatología de la agresividad, encontrándose vinculaciones entre neurotransmisores y agresividad. Este enfoque sería igualmente reduccionista como el de Lorenz, al encontrar como causa central de la agresividad alteraciones sólo de orden biológico. Un atenuante a esta postura biologista, es la cautela de los investigadores, al admitir todo lo que aún se ignora sobre los mecanismos productores de la agresividad.

Como contrapartida a las teorías biológicas, los modelos interactivos ponen énfasis en la agresividad como una disfunción de las habilidades sociales y en cuya estructuración parecieran estar implicados factores individuales, de contexto $y$, esencialmente, la interacción entre ambos. A su vez, los desarrollos de la psicología cognitiva han brindado elementos para analizar en qué fase del proceso de la comunicación se produce una distorsión en cómo se procesa la información, esto es, como se analiza e interpreta el mensaje que se recibe, de tal manera que 
genera como respuesta una agresión. También resultan útiles los conceptos de la teoría de la frustración- agresión, desde cuya concepción el comportamiento agresivo emerge cuando el sujeto ve bloqueadas o interferidas las posibilidades de lograr una meta. De los modelos interactivos, se considera como relevante a la teoría socio cognitiva de Bandura.

El interés de Bandura es poder explicar la adquisición de nuevos comportamientos sociales, en especial el agresivo en niños; estudia así el papel de la imitación, del modelado, del aprendizaje por observación y las pautas de refuerzo en la adquisición de comportamientos socialmente aceptables, o bien censurados. Pone mucho mayor énfasis en las variables sociales que las teorías pre-existentes. Las críticas a esta teoría se centran en que opera sobre el comportamiento manifiesto, dejando de lado emociones y conflictos humanos, que podrían explicar por qué el modelado no es igualmente exitoso en todos los sujetos.

\section{Alcances y perspectivas acerca de los estudios sobre agresividad}

Las diversas teorías analizadas han permitido bucear con mayor profundidad en las causas del comportamiento agresivo, pero tienen la limitación de ser una mirada parcial. El fenómeno de la agresividad en aumento da cuenta de un malestar en consonancia con la complejidad de las sociedades actuales; malestar que resulta más notorio en la adolescencia por ser una etapa de transformaciones en la personalidad y de definición de la identidad. Así también puede decirse que se necesita un mayor refinamiento de las teorías, que se interroguen acerca del alcance de las mismas y del significado supuestamente universal que se atribuye a determinados comportamientos categorizados como agresivos; igualmente es preciso contar con instrumentos de evaluación y diagnóstico más fiables, que operacionalicen el constructo agresividad y permitan diferenciarlo de otros conexos, como el de violencia. Todo ello haría posible, en una relación dialéctica teoría-práctica, precisar de qué se está hablando cuando se alude a agresividad. Por otro lado, faltan aún estudios que comparen, con rigor metodológico, el comportamiento agresivo en el adolescente y en el adulto que arrojen luz acerca de la prevalencia de la emergencia de la agresividad en uno y otro grupo etario. Estas investigaciones permitirían esclarecer hasta qué punto se intensifican en la adolescencia. En las prácticas psicológicas del siglo XX hubo una tendencia a estigmatizar esta etapa del ciclo vital, con etiquetas asociadas a la psicopatología y el déficit; sin embargo nuevos estudios ponen en duda esta perspectiva, al no encontrar diferencias significativas entre unos y otros (adolescentes y adultos).

Por último, cabe señalar que en la actualidad las intervenciones derivadas solo de algunas teorías resultan parciales y, por ende insuficientes, habida cuenta 
de que la problemática, lejos de decrecer, va en constante aumento. Constituye un reto lograr una mayor integración entre dichas teorías que permita obtener una perspectiva multidimensional de la problemática y que conduzcan a intervenciones más eficaces. De este modo sería posible pasar de la agresividad a las habilidades sociales saludables, recurso indispensable para la inclusión del sujeto en su grupo de referencia.

\section{Referencias}

Achenbach, T. (2008). Assessment, diagnosis, nosology and taxonomy of child and adolescent psychopathology. In Hersen \& Gross (Eds.) Handbook of clinical psychology, NY, US: John Wiley \& Sons Inc.

Achenbach, T. \& Edelbrock, C. (1979). The child behavior profile: II. Boys aged 12-16 and girls aged 6-11 and 12-16. Journal of Consulting and Clinical Psychology, 2, 223-233.

Andreu, J., Ramírez, J. \& Raine, F. (2006). Un modelo dicotómico de la agresión: valoración mediante dos auto-informes (CAMA y RPQ). Psicopatología Clínica Legal y Forense, 5, 25-42.

American Psychiatric Association (2014). Guía de Consulta de los criterios diagnósticos del DSM 5. Madrid: Editorial Médica Panamericana.

Bandura, A. (2001). Social Cognitive Theory; An Agentic perspective. Annual Review of Psychology, 52, 1-26.

Bandura, A. \& Locke, E. (2003). Negative Self-Efficacy and Goal Effects Revisited. Journal of Applied Psychology, 88(1), 87-99.

Bandura, A. \& Walters, R. (1974). Aprendizaje social y desarrollo de la personalidad. Madrid: Alianza Universidad.

Buss, A. H. (1961). The Psychology of Aggression. New York: Wiley.

Buss, A. \& Perry. M. (1992). The aggression questionnaire. Journal of Personality and Social Psychology, 63, 452-459.

Caballo, V. (2007). Manual de evaluación y entrenamiento de las habilidades sociales (7 a. ed.). Madrid: Siglo XIX. 
Carrasco Ortiz, M \& González Calderón, M. (2006). Aspectos conceptuales de la agresión: definición y modelos explicativos. Acción Psicológica, 4(2), 7-38.

Contini, N. (2009). Las habilidades sociales en la adolescencia temprana: perspectivas desde la psicología positiva. Psicodebate. Universidad de Palermo, 9, 45-64.

Crick, N. \& Dodge, K. (1996). Social Information-processing mechanisms in reactive and proactive aggression. Child Development, 67, 710-722.

Davidson, R., Putnam, K. \& Larson, C. (2000). Disfunction in the Neural Circuity of Emotion Regulation--A Possible Prelude to Violence. Science, 289(5479), 591-594.

Del Prette, Z., Teodoro, M. \& Del Prete, A. (2014). Habilidades socias de adolescentes: validade convergente entre o IHSA-Del-Prette e a MESSY. Estudos de Psicología Campinas, 31(1), 15-23. doi: 10.1590/0103166X2014000100002

Dodge, K. \& Crick, N. (1990). Social information processing bases of aggressive behaviour in children. Personality and Social Psychology Bulletin, 15, 8-22.

Dollard, J., Doob, L, Miller, N, Mowerer, O. \& Sears, R. (1939). Frustration and aggression. New Haven, Conn.: Yale University Press.

Garaigordobil Landazabal, M. (2008). Assessment of the effects of a cooperative play programmed for children aged 10-11 years on social adaptation and on the perception that parents, teachers and peers have of children's prosocial behaviors. Infancia y Aprendizaje: Journal for the Study of Education and Development, 31(3), 303-318.

Garaigordobil, M. \& Oñederra, J. (2010). Teorías explicativas de la agresividad y la conducta violenta. En M. Garaibordobil y J. Oñederra (Eds.), La violencia entre iguales. Revisión teórica y estrategias de intervención (pp. 65-89). Madrid: Pirámide.

Gil, F. \& León Rubio, J. (1998). Habilidades Sociales. Teoría, investigación e intervención. Madrid: Síntesis.

Halsband, S. \& Barenbaum, R. (2008). Neurobiología de la agresividad. Papel del litio y los antidepresivos (pp. 19-32). En S. Halsband (Ed.) Agresividad. Buenos Aires: Polemos. 
Imaz Roncero, C., Gonzales Gallegos, K., Geijo Uribe, M , Higuera González, M., \& Sánchez Lorenzo, I. (2013). Violencia en la adolescencia. Pediatría Integral, XVII(2), 101-108.

Inglés, C., Benavides, G., Redondo, J., García-Fernández, J., Ruiz-Esteban, C., Estévez, C., \& Huescar, E. (2009). Conducta prosocial y rendimiento académico en estudiantes españoles de Educación Secundaria Obligatoria. Anales de Psicología, 25(1), 93-101.

Kohn, M. (1977). Social competence, symptoms and underachievement in childhood. A longitudinal perspective. Nueva York: Holt, Rinchart and Winston

Laplanche, J. \& Pontalis, J. (2009). Diccionario de Psicoanálisis (11 reimpresión de la $1^{\circ}$ edición). Buenos Aires: Paidós,.

Leon Rubio, J. \& Medina Anzano, S. (1998) Aproximación conceptual a las habilidades sociales. En F. Gil \& J. León Rubio (Eds.), Habilidades sociales, Teoría, Investigación e Intervención. Madrid: Síntesis.

Lorenz, K. (1971). Biología del comportamiento. México: Siglo XXI Editores S.A.

Lorenz, K. (1998). Sobre la agresión: el pretendido mal. México: Siglo XXI Editores, $20^{\circ}$ Edición. Primera edición en español 1971.

McFall, R. (1982) A review and reformulation of the concept of social skills. Behavioural Assessment, 4, 1-33.

Martorell, M. (1997). Evaluación de la conducta social. En M. Casullo (Ed.) Evaluación psicológica en el campo socioeducativo. Buenos Aires. Paidós.

Monjas Casares, M. (2000). Programa de enseñanza de habilidades de interacción social (PEHIS) para niños y niñas en edad escolar. Madrid: CEPE.

Organización Mundial de la Salud (1999). Trastornos mentales y del comportamiento de la décima revisión de la Clasificación Internacional de las Enfermedades CIE-10. Madrid: Editorial Médica Panamericana.

Osofsky, J. (1999). The impact of violence on children. The future of Children, 9(3), 33-49.

Pakaslahti, L. (2000). Childrens' and adolescents' aggressive behaviour in context: the development and application of aggressive problem-solving 
strategies. Aggression and Violent Behaviour, 5, 467-490.

Real Academia Española (1992). Diccionario de la Lengua Española (21ra edición). RAE: Madrid.

Schultz, D. \& Schultz, S. (2010). Teorías de la personalidad. (9na. edición) México: Cengage learning.

Silva, F., Moro, M., \& Generós Ortet, G. (1997). Dimensiones de orientación interpersonal: Un modelo y un instrumento. En M. Garaigordobil Landazábal \& C. Maganto (Eds.), Socialización y conducta prosocial en la infancia y en la adolescencia (pp. 89-108). San Sebastián: Servicio editorial de la Universidad del País Vasco.

Spielberger, C., Jacobs, G., Russell, S. \& Crane, R. (1983). Assessment of Anger: the State-Trait Anger Scale. En J. Butcher \& C. Spielberger (Eds.), Advances in Personality Assessment, (Vol. 2, pp. 157-187) . Hillsdale: LEA.

Swann, A. (2003), Neuroreceptor Mechanisms of aggression and its Treatment. Journal of Clinical Psychiatry, 64(4), 26-35.

Trower, P. (1986). Social skills training and social anxiety. En C. Hollin \& P. Trower (Eds.), Handbook of social skills training. Oxford: Pergamon Press. 
